\title{
HD 152246: a new high-mass triple system and its basic properties ${ }^{\star}$
}

\author{
A. Nasseri ${ }^{1}$, R. Chini ${ }^{1,2}$, P. Harmanec ${ }^{3}$, P. Mayer ${ }^{3}$, J. A. Nemravováa ${ }^{3}$, T. Dembsky ${ }^{1}$, H. Lehmann ${ }^{4}$, \\ H. Sana ${ }^{5}$, and J.-B. Le Bouquin ${ }^{6}$
}

\author{
1 Astronomisches Institut, Ruhr-Universität Bochum, Universitätsstr. 150, 44801 Bochum, Germany \\ e-mail: chini@astro.rub.de \\ 2 Instituto de Astronomía, Universidad Católica del Norte, Avenida Angamos 0610, Casilla 1280 Antofagasta, Chile \\ 3 Astronomical Institute of the Charles University, Faculty of Mathematics and Physics, V Holešovičkách 2, 18000 Praha 8, \\ Czech Republic \\ 4 Thüringer Landessternwarte Tautenburg, 07778 Tautenburg, Germany \\ 5 ESA/Space Telescope Science Institute, 3700 San Martin drive, MD 21218, Baltimore, USA \\ ${ }^{6}$ Institut d'Astrophysique et de Planétologie de Grenoble, CNRS-UJF UMR 5571, 414 rue de la Piscine, 38400 St-Martin-d'Hères, \\ France
}

Received 12 June 2014 / Accepted 25 July 2014

\begin{abstract}
Analyses of multi-epoch, high-resolution $(R \sim 50000)$ optical spectra of the O-type star HD 152246 (O9 IV according to the most recent classification), complemented by a limited number of earlier published radial velocities, led to the finding that the object is a hierarchical triple system, where a close inner pair $(\mathrm{Ba}-\mathrm{Bb})$ with a slightly eccentric orbit $(e=0.11)$ and a period of 6.0049 revolves in a 470-day highly eccentric orbit $(e=0.865)$ with another massive and brighter component $\mathrm{A}$. The mass ratio of the inner system must be low since we were unable to find any traces of the secondary spectrum. The mass ratio $\mathrm{A} /(\mathrm{Ba}+\mathrm{Bb})$ is 0.89 . The outer system has recently been resolved using long-baseline interferometry on three occasions. The interferometry confirms the spectroscopic results and specifies elements of the system. Our orbital solutions, including the combined radial-velocity and interferometric solution indicate an orbital inclination of the outer orbit of $112^{\circ}$ and stellar masses of 20.4 and $22.8 M_{\odot}$. We also disentangled the spectra of components $\mathrm{A}$ and $\mathrm{Ba}$ and compare them to synthetic spectra from two independent programmes, TLUSTY and FASTWIND. In either case, the fit was not satisfactory and we postpone a better determination of the system properties for a future study, after obtaining observations during the periastron passage of the outer orbit (the nearest chance being March 2015). For the moment, we can only conclude that component A is an O9 IV star with $v \sin i=210 \pm 10 \mathrm{~km} \mathrm{~s}^{-1}$ and effective temperature of $33000 \pm 500 \mathrm{~K}$, while component $\mathrm{Ba}$ is an $09 \mathrm{~V}$ object with $v \sin i=65 \pm 3 \mathrm{~km} \mathrm{~s}^{-1}$ and $T_{\text {eff }}=33600 \pm 600 \mathrm{~K}$.
\end{abstract}

Key words. binaries: spectroscopic - stars: massive - stars: fundamental parameters - stars: individual: HD 152246

\section{Introduction}

The only stable configuration of a stellar triple system seems to consist of a close binary and a substantially more distant third component. These systems are important for testing theories of star formation and stellar evolution in the presence of nearby companions.

The source HD 152246 (HIP 82685) is a member of the Sco OB1 association at a distance of about $1.585 \mathrm{kpc}$ (Sung et al. 2013). Although the star has been included in many photometric and spectroscopic surveys of $\mathrm{O}$ stars, the knowledge of its properties is quite limited.

The binary nature of HD 152246 was discovered by Thackeray et al. (1973) who published six radial velocities (RVs) from 1963-1967 showing a range of about $120 \mathrm{~km} \mathrm{~s}^{-1}$, but this finding has not received much attention. Conti et al. (1977)

* Based on data products from observations made with ESO telescopes at La Silla Paranal Observatory under programmes 68.D-0095(A), 71.D-0369(A), 073.D-0609(A), 075.D-0061(A), 076.D0294(A)， 077.D-0146(A)， 079.D-0718(A)， 081.D-2008(B), 083.D-0589(B), 086.D-0997(B), 087.D-0946(A), and 089.D-0975(A), extracted from the ESO/ST-ECF Science Archive Facility, and on the BESO spectra. published one new RV and noted that the star is a RV variable. Penny (1996) suspected the presence of a weak secondary in the CCF peaks, but it was only Stickland \& Lloyd (2001) who discovered the presence of sharp and wide lines in the IUE spectra and published two RVs for both components. They interpreted the two systems of lines as belonging to the primary and secondary in a binary system. Mason et al. (1998) observed the star with speckle interferometry, but could not detect any companion for the angular separation range 0 .' $^{\prime} 035<\rho<11^{\prime \prime} 5$ with $\Delta m<3$.

The HD 152246 system has been a part of our monitoring programme for stellar multiplicity (Chini et al. 2012) and attracted our attention because of its varying spectral line profiles. In our preliminary report based on an analysis of the He I $5876 \AA$ line (Nasseri et al. 2013) we showed that HD 152246 must be a hierarchical triple system. What is actually observed is a combination of a broad-line O-type star (component A) and another O-type star with narrow lines, which itself is the primary component $\mathrm{Ba}$ of a close pair. The RVs of the narrow-line star follow the orbital motion with an invisible secondary $\mathrm{Bb}$ with a period of about $6 \mathrm{~d}$ and also a motion around the common centre of gravity with the component $\mathrm{A}$. The RVs of the broad-lined component A follow only the orbital motion in the wide orbit (we 
Table 1. Previously published determinations of the projected rotational velocity of HD 152246.

\begin{tabular}{cl}
\hline \hline$v \sin i\left(\mathrm{~km} \mathrm{~s}^{-1}\right)$ & Source \\
\hline 280 & Conti \& Ebbets (1977) \\
$90 \pm 20$ & Grigsby et al. (1992) \\
103 & Penny (1996) \\
72 & Howarth et al. (1997) \\
59 and 156 & Stickland \& Lloyd (2001) \\
\hline
\end{tabular}

had tentatively derived a period of 53 days for it; we revise the period here).

The combined spectral type of HD 152246 was first determined by Morgan et al. (1955) as O9: III.. Later, more accurate but fairly similar properties were found: Schild et al. (1969) and Garrison et al. (1977) classified the star as O9 III while Walborn (1973) and Thackeray et al. (1973) assigned spectral types of O9 III-IV(n) and O8.5 III, respectively. Several published measurements of the visual brightness of HD 152246 are mutually quite consistent (Thackeray et al. 1973; Klare \& Neckel 1977; Dachs et al. 1982; Schild et al. 1983; Hog et al. 2000). Mermilliod \& Mermilliod (1998) give the following weighted mean value $V=7 \mathrm{~m} .308 \pm 0$. 015 . The only deviating value was reported by Heske \& Wendker (1984): $V=8$ m 09 , which we suspect could be a misidentification of the star. We also used all HIPPARCos $H_{\mathrm{p}}$ observations with flags 0 and 1 (Perryman \& ESA 1997) and transformed them to Johnson $V$ following Harmanec (1998) to obtain $V=7.330 \pm 0.008$. The full range of individual values is 0 . 05 , so a future test for micro-variability would perhaps be useful. In any case it seems that no binary eclipses are observed.

Several - seemingly contradictory - determinations of the projected rotational velocity of HD 152246 were published (see Table 1). It seems clear now that Conti \& Ebbets (1977) probably derived $v \sin i$ from the unresolved blend of lines of both stars, other investigators mainly measured the width of the narrow component Ba until Stickland \& Lloyd (2001) resolved and measured the lines of both spectra.

In this paper we present the analysis of 49 high resolution spectra, obtain rather accurate orbital elements and estimate the basic physical properties of the system.

\section{Observations and data reduction}

\subsection{Spectroscopy}

We have secured 49 high resolution $(R \sim 50000)$ echelle spectra covering the wavelength range from 3620 to $8530 \AA$ and the time interval from May 1999 to October 2013. Twenty-six spectra were taken at the ESO with the spectrograph FEROS (RJDs 51 327.9-56098.8); the first two spectra from March and April 2002 stem from the ESO $1.52 \mathrm{~m}$ telescope, the remaining data come from the MPG/ESO $2.2 \mathrm{~m}$ telescope. Twenty-three spectra were obtained with BESO (RJDs 54913.9-56585.5) ${ }^{1}$ at the Universitätssternwarte Bochum on a side-hill of Cerro Armazones in Chile. BESO (Fuhrmann et al. 2011) is a clone of the ESO spectrograph FEROS on La Silla and has been attached to the $1.5 \mathrm{~m}$ Hexapod-Telescope. The identical spectrographs allowed a perfect combination of the two data sets. All spectra were reduced with a pipeline based on a MIDAS package

\footnotetext{
1 Throughout this study we shall use the following abbreviation for the reduced Julian date $\mathrm{RJD}=\mathrm{HJD}-2400000.0$.
}

Table 2. Published and new RVs of components A and Ba.

\begin{tabular}{|c|c|c|c|}
\hline RJD & $\mathrm{RV}_{\mathrm{A}}$ & $\mathrm{RV}_{\mathrm{Ba}}$ & Source \\
\hline 38251.2378 & - & 36.0 & Thackeray et al. (1973) \\
\hline 39200.6408 & - & 60.0 & Thackeray et al. (1973) \\
\hline 39200.6498 & - & 52.0 & Thackeray et al. (1973) \\
\hline 39688.3284 & - & -18.0 & Thackeray et al. (1973) \\
\hline 39720.2631 & - & -58.0 & Thackeray et al. (1973) \\
\hline 39723.2539 & - & 50.0 & Thackeray et al. (1973) \\
\hline 42888.78 & - & -29.8 & Conti et al. (1977) \\
\hline 44995.778 & -109.4 & 46.6 & Stickland \& Lloyd (2001) \\
\hline 45000.609 & -103.0 & 26.5 & Stickland \& Lloyd (2001) \\
\hline 51327.8888 & -7.0 & -50.8 & this paper, FEROS \\
\hline 52040.8836 & -49.0 & -20.5 & this paper, FEROS \\
\hline 52338.8394 & -23.0 & 8.6 & this paper, FEROS \\
\hline 52383.7834 & -25.0 & -44.3 & this paper, FEROS \\
\hline 52783.6903 & -15.0 & 2.4 & this paper, FEROS \\
\hline 52784.6323 & -19.0 & -28.5 & this paper, FEROS \\
\hline 53130.7748 & 44.0 & -58.5 & this paper, FEROS \\
\hline 53131.6933 & 37.0 & -39.6 & this paper, FEROS \\
\hline 53132.7522 & 42.5 & -64.3 & this paper, FEROS \\
\hline 53133.7812 & 37.0 & -94.0 & this paper, FEROS \\
\hline 53134.7494 & 34.0 & -101.1 & this paper, FEROS \\
\hline 53135.7069 & 33.0 & -81.9 & this paper, FEROS \\
\hline 53547.6424 & -73.0 & 8.3 & this paper, FEROS \\
\hline 53798.9178 & -30.0 & 9.2 & this paper, FEROS \\
\hline 53862.8912 & -41.0 & -1.4 & this paper, FEROS \\
\hline 53865.8991 & -40.5 & -14.2 & this paper, FEROS \\
\hline 54212.8182 & -26.0 & 12.4 & this paper, FEROS \\
\hline 54627.7027 & -5.0 & -12.8 & this paper, FEROS \\
\hline 54976.7046 & -42.0 & -7.2 & this paper, FEROS \\
\hline 55641.8195 & -18.5 & 22.5 & this paper, FEROS \\
\hline 55642.8300 & -19.0 & -9.4 & this paper, FEROS \\
\hline 55696.8448 & -23.0 & -0.7 & this paper, FEROS \\
\hline 55697.7549 & -22.0 & -35.6 & this paper, FEROS \\
\hline 55698.8283 & -21.0 & -48.3 & this paper, FEROS \\
\hline 56068.7356 & -17.5 & 3.9 & this paper, FEROS \\
\hline 56098.8047 & -14.0 & 4.8 & this paper, FEROS \\
\hline 54913.8587 & -48.0 & 20.3 & this paper, BESO \\
\hline 54920.8675 & -68.0 & 46.3 & this paper, BESO \\
\hline 55050.5851 & 18.5 & -82.1 & this paper, BESO \\
\hline 56485.5223 & 1.5 & -78.8 & this paper, BESO \\
\hline 56490.6158 & -1.0 & -72.8 & this paper, BESO \\
\hline 56495.6219 & -4.0 & -31.9 & this paper, BESO \\
\hline 56498.7118 & -10.0 & -45.9 & this paper, BESO \\
\hline 56502.5216 & -10.0 & -65.2 & this paper, BESO \\
\hline 56508.4660 & -3.5 & -62.4 & this paper, BESO \\
\hline 56512.5388 & -3.5 & 9.9 & this paper, BESO \\
\hline 56516.5365 & -12.0 & -48.3 & this paper, BESO \\
\hline 56518.6466 & -10.5 & 11.4 & this paper, BESO \\
\hline 56521.5196 & -13.0 & -71.1 & this paper, BESO \\
\hline 56532.5305 & -24.0 & -59.7 & this paper, BESO \\
\hline 56540.5434 & -18.5 & -43.1 & this paper, BESO \\
\hline 56541.5275 & -13.0 & -4.0 & this paper, BESO \\
\hline 56542.5155 & -13.0 & 15.4 & this paper, BESO \\
\hline 56545.4841 & -12.0 & -62.6 & this paper, BESO \\
\hline 56546.5149 & -13.0 & -39.5 & this paper, BESO \\
\hline 56547.5287 & -21.0 & -0.8 & this paper, BESO \\
\hline 56548.5231 & -20.5 & 17.1 & this paper, BESO \\
\hline 56576.4898 & -20.0 & -43.7 & this paper, BESO \\
\hline 56585.4939 & -20.0 & -5.5 & this paper, BESO \\
\hline
\end{tabular}

Notes. The new velocities are based on the fit of the He I $5876 \AA$ line by a combination of a Gaussian and a rotationally broadened profile (see text for details). 


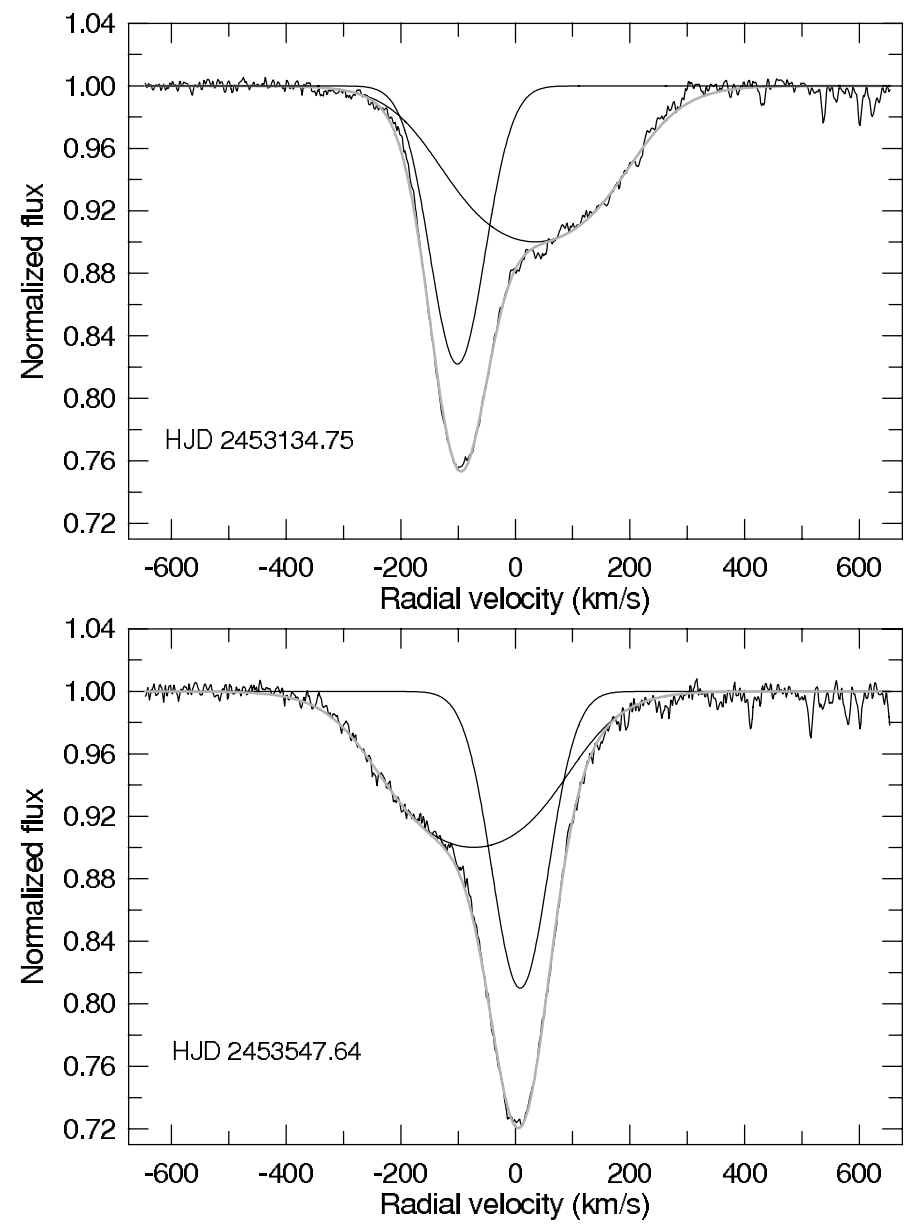

Fig. 1. Two He I $5876 \AA$ A line profiles from different orbital phases. The observed profiles have been fitted by a narrow Gaussian and a wide rotationally broadened profile. The resulting fits are denoted by grey curves.

adapted from FEROS. The signal-to-noise ratio of the reduced FEROS spectra ranges from 247 to 487; that of the BESO spectra from 65 to 177. For either instrument, the signal-to-noise ratio was measured in the neighbourhood of the He I line at $5876 \AA$.

At a later stage of the analysis, we also used 9 published RVs of component $\mathrm{B}$ and provided a few comments on 2 published RVs of component A (Table 2).

\subsection{Long-baseline interferometry}

Interferometric measurements of HD 152246 were obtained on February 24, April 4 and May 8, 2014 using the PIONIER fourbeam combiner (Le Bouquin et al. 2011) at the four Auxiliary Telescopes of the ESO Very Large Telescope Interferometer (VLTI, Haguenauer et al. 2008, 2010). The data were reduced with the pndrs package (Le Bouquin et al. 2011). Reduction steps and achieved calibration accuracy on the O-type stars were described earlier (Sana et al. 2013) and will not be repeated here. The data analysis closely follows the procedure used in the Southern Massive Star at High angular resolution survey (Sana et al. 2014), which uniformly analyses PIONIER observations of a sample of over 100 O-type stars.

HD 152246 was clearly resolved at three epochs with a separation of about 3.2-3.3 milli-arcsec (mas). A clear rotation of the binary axis could be detected over the 73 day baseline of our
PIONIER observations. Table 3 provides the journal of the interferometric observations together with the parameters of the best fit binary model. Figure 2 shows and example of the measured data and the best fit binary model.

\section{Line profiles, radial velocities and orbital periods}

To have a guidance to a more sophisticated analysis, we first measured RVs of both components of the strong and usually well-exposed He I $5876 \AA$ line, fitting the narrow component by a Gaussian and the broader one by a rotationally broadened profile. The examples of such fits are shown in Fig. 1 and all RVs, together with nine RVs from the literature, are in Table 2.

We note that Nasseri et al. (2013) originally reported a tentative value of 53 days for the longer period and 6 days for the shorter period. Meanwhile we could recover a number of old spectra from the epoch 1999-2003 which led to a substantial revision of the originally suggested long period.

The analysis of the RV variations soon showed that the RV of the component A varies with a single period, which turned out to be about $470 \mathrm{~d}$. The RVs of component $\mathrm{Ba}$ are modulated also by this period (showing anti-phase variations with respect to those of component A), but they also varied with a shorter period of about $6 \mathrm{~d}$. After deriving a more accurate value of the short period, treating data subsets as having individual systemic velocities (to allow for the changes with the $470 \mathrm{~d}$ orbit), we included all RVs and derived an orbital solution for the triple system. For this task the programme FOTEL (Hadrava 2004a, and references therein) is well suited. We also verified that the $6 \mathrm{~d}$ period is clearly seen in the RVs for the narrow component published by Thackeray et al. (1973), Conti et al. (1977), and Stickland \& Lloyd (2001). The situation is a bit more complicated with the two RVs for component $\mathrm{A}$ as we shall discuss below.

In a second step, we therefore derived a solution for the triple-star system with FOTEL, using all Gaussian RVs for both components measured in our new spectra and also all 9 RVs of component B from the literature, first with equal weights and then weighting the three data subsets by the weights inversely proportional to the square of root-mean-square residuals (rms) from the preliminary solution. This solution is presented in Table 4. The RV curve for the $6 \mathrm{~d}$ orbit is shown in Fig. 3 and corresponds to Table 4 . The curve for the $470 \mathrm{~d}$ orbit is presented in Fig. 4; the parameters based also on the interferometric results as listed in Table 6 were used for its construction.

Stickland \& Lloyd (2001) published two RVs for the component A (see Table 2):

$-109.4 \mathrm{~km} \mathrm{~s}^{-1}$ at RJD 44995.7780 , phase 0.7811 , and

$-103.0 \mathrm{~km} \mathrm{~s}^{-1}$ at RJD 45000.6090 , phase 0.7913 .

It is clear from Fig. 3 (bottom panel) that these RVs are for some $-50 \mathrm{~km} \mathrm{~s}^{-1}$ too negative than our new RVs. The difference could be made smaller if the true value of the long period would be $474.3 \mathrm{~d}$. However, this change in the period would increase the rms of the orbital solution of component B to $5.6 \mathrm{~km} \mathrm{~s}^{-1}$, and even then, a deviation of about $30 \mathrm{~km} \mathrm{~s}^{-1}$ would persist. One can conjecture that the reason for too negative RVs measured by Stickland \& Lloyd (2001) lies in that our results (see below) show that $v \sin i$ of component $\mathrm{A}$ is about $200 \mathrm{~km} \mathrm{~s}^{-1}$, while they only derived $156 \mathrm{~km} \mathrm{~s}^{-1}$. As the relation between the narrow and broad profiles measured by Stickland \& Lloyd (2001) was similar to that in Fig. 1 (bottom panel) here, the smaller $v \sin i$ means that the RVs of broad profiles as measured by them might be erroneously shifted by about one half of the $v \sin i$ difference, 

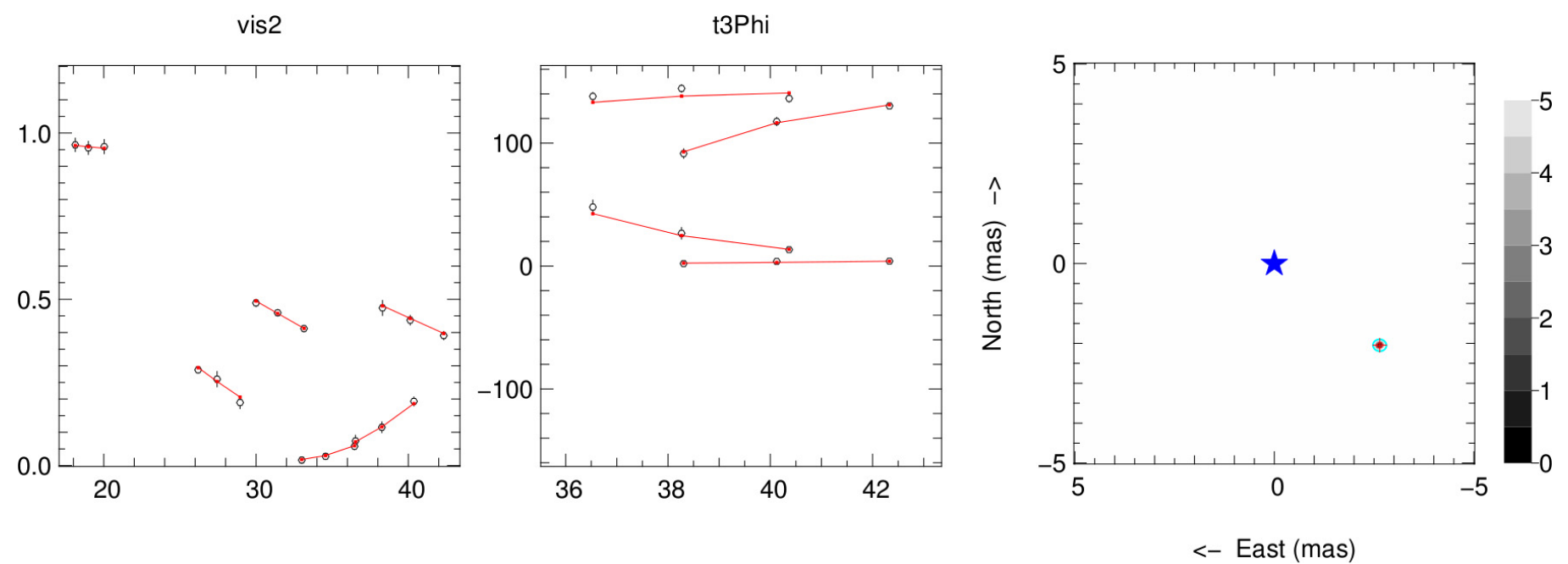

Fig. 2. Visibilities (left panel), closure phase (middle panel) and $\chi^{2}$ map of the Feb 28 PIONIER observation of HD 152246 . The best-fit binary model is overlaid.

Table 3. Interferometric best-fit measurements and $1 \sigma$ error-bars.

\begin{tabular}{lcccc}
\hline \hline & & \multicolumn{3}{c}{ Observing date } \\
Parameter & Unit & 2014-Feb.-24 & 2014-Apr.-04 & 2014-May-08 \\
\hline RJD & & 56712.840 & 56751.778 & 56785.902 \\
$\left(f_{\mathrm{B}} / f_{\mathrm{A}}\right)_{1.65 \mu}$ & & $0.77 \pm 0.01$ & $0.76 \pm 0.04$ & $0.79 \pm 0.1$ \\
$\delta \mathrm{E}$ & $(\mathrm{mas})$ & $-2.64 \pm 0.17$ & $-2.23 \pm 0.13$ & $-1.72 \pm 0.19$ \\
$\delta \mathrm{N}$ & $($ mas) & $-2.05 \pm 0.15$ & $-2.23 \pm 0.07$ & $-2.25 \pm 0.26$ \\
$r$ & $(\mathrm{mas})$ & $3.34 \pm 0.16$ & $3.15 \pm 0.10$ & $2.83 \pm 0.24$ \\
$\theta$ & $\left({ }^{\circ}\right)$ & $232.2 \pm 1.6$ & $224.9 \pm 0.2$ & $217.4 \pm 4.5$ \\
$\chi_{\text {red }}^{2}$ & & 0.6 & 0.9 & 0.8 \\
\hline
\end{tabular}

i.e. more than by $20 \mathrm{~km} \mathrm{~s}^{-1}$. Such a shift could explain the observed RV difference. The ultimate solution to this problem as well as the discrimination between a number of possible values of $K_{\mathrm{A}}$ might come only with future dedicated observations, densely covering the phases of the periastron passage (the first chance being in March 2015). In any case, it is clear that the two RVs by Stickland \& Lloyd (2001) provide additional support for the $470 \mathrm{~d}$ period.

\section{RV orbital solutions with KOREL}

The FOTEL orbital elements were eventually used as initial values for the solution with the spectral-disentangling programme KOREL (Hadrava 2004b). Apart from the He I $5876 \AA$ line, we used five other suitable lines and spectral segments (He I 6678, He I 7065, He II 5411, O III 5592, and a longer segment 5670-5835 ̊, which contains C III 5695, C IV 5801, and C IV $5812 \AA$ plus several stronger diffuse interstellar bands) to obtain six independent KOREL solutions.

In practice, we searched the parameter space and ran a number of trial solutions for each spectral region to find out a solution with the lowest sum of residuals. These final solutions and the resulting elements are summarised in Table 5 together with their mean values. We note that KOREL does not provide error estimates so the comparison of several independent solutions gives us some idea about the possible errors of the elements.

We carried out several attempts to detect also some weak lines of component $\mathrm{Bb}$ in KOREL solution but with no result. This means that we are unable to obtain the mass ratio between components $\mathrm{Ba}$ and $\mathrm{Bb}$ and conclude that the component $\mathrm{Bb}$ is much less massive than component $\mathrm{Ba}$. This is similar to HD 165246 binary, for which Mayer et al. (2013) found a low mass ratio of 0.17 .

\section{Three-dimensional orbit of HD 152246 A, B}

Having resolved the A,B pair and detected its orbital motion on the plane of the sky opens the possibility of computing the threedimensional orbit of HD 152246. To this aim, we simultaneously fit the RV and astrometric measurements using the method described in Sana et al. (2013); we only used FEROS and BESO data. We corrected the RVs of component $\mathrm{Ba}$ listed in Table 2 from the orbital motion around the centre of mass of the $\mathrm{Ba}, \mathrm{Bb}$ pair using the orbital solution given in Table 4. We adopted error bars on the A and B RV components given by the residual of the fit in Table 2, i.e. 5.66 and $4.29 \mathrm{~km} \mathrm{~s}^{-1}$ for component A and B, respectively. We also neglected the astrometric displacement of $\mathrm{B}$ attributable to its close companion in the VLTI data. This is a valid assumption given such displacement is likely of the order of 0.1 mas.

We used the averaged KOREL orbital solution as a starting point for our fit. We fixed the distance at $1585 \mathrm{kpc}$ and we worked in the systemic velocity frame (i.e., $\gamma=0 \mathrm{~km} \mathrm{~s}^{-1}$ ). The obtained best-fit solution is given in Table 6 and shown in Fig. 4. As explained in Sana et al. (2013), we estimated the uncertainties on the best-fit parameters by Monte Carlo simulations. We randomly drew 1000 artificial RV and astrometric data sets. We preserved the observational time sampling. The data points were taken from Gaussian distributions centred on the best fit solution, using the observational error bars as the Gaussian standard deviations to draw. We then ran the fitting procedure on each 

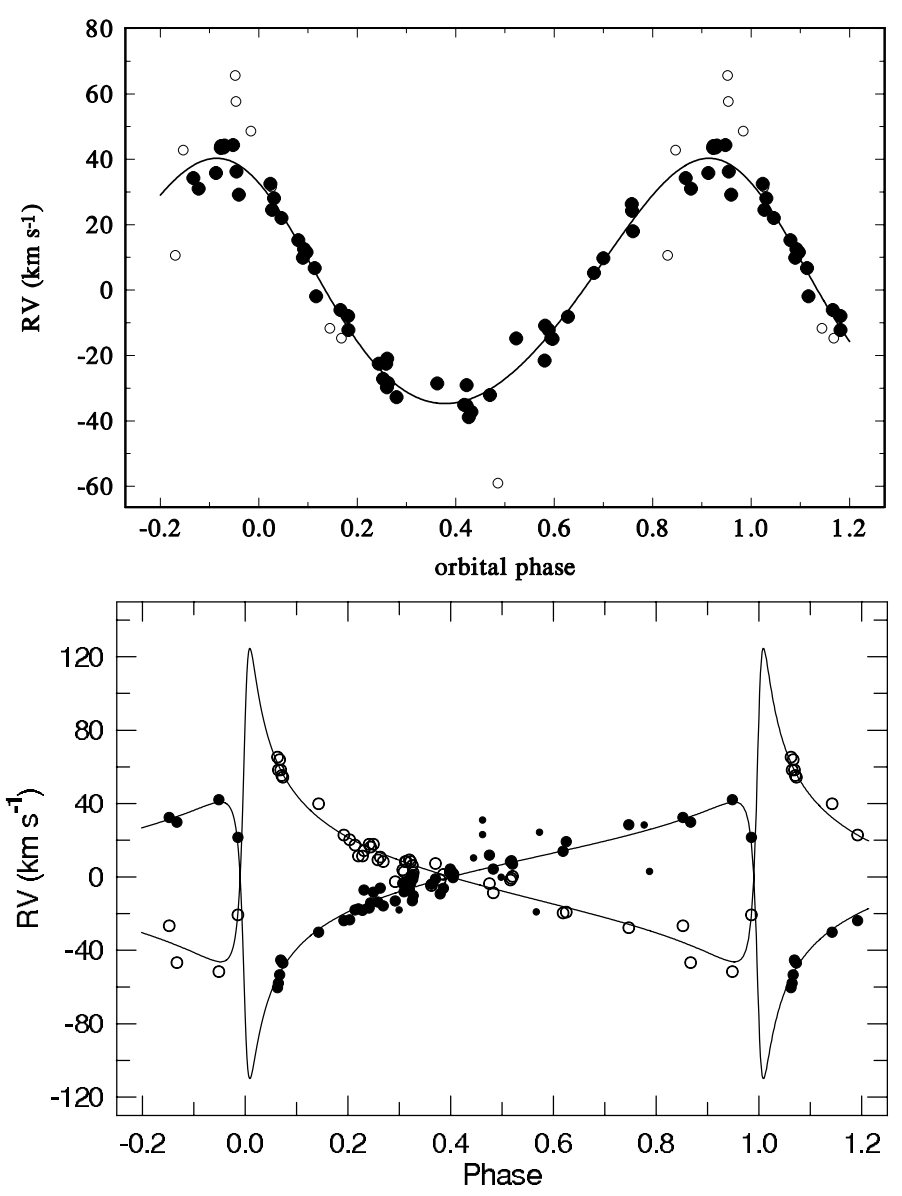

Fig. 3. Phase plots for both binary orbits. Top panel: RVs of component $\mathrm{Ba}$, prewhitened for the variations in the $470 \mathrm{~d}$ orbit and plotted vs. phase for the $6 \mathrm{~d}$ period and periastron epoch from Table 4. Open circles denote RVs from the literature. Bottom panel: RV curves of components $\mathrm{A}$ and $\mathrm{B}$ in the wide orbit. Open circles denote component $\mathrm{A}$, filled circles component $\mathrm{B}$ (smaller ones for RVs from the literature). We note that in both plots, RVs $-\gamma$ are plotted.

of the synthetic data set and we estimated the uncertainties on the best-fit parameters from the distribution of retrieved parameters. The error intervals quoted in Table 6 correspond to the $68 \%$ confidence intervals. The intervals are taken as symmetric if the upper and lower error bars do not differ by more than $10 \%$, asymmetric confidence intervals are otherwise provided.

Comparing the results of the three-dimensional orbit with the FOTEL and KOREL orbital solutions (Tables 4 and 5) reveal excellent agreement. The preference for a high eccentricity value is confirmed while we obtained slightly smaller RV semi-amplitudes. Importantly, the orbital inclination $i$ is now constrained to $i=112.46_{-9.11}^{+6.98}$. This allows us to derive absolute masses for the $\mathrm{A}$ and $\mathrm{B}$ components to $20.35 \pm 1.50$ and $22.78 \pm 1.82 M_{\odot}$, i.e. with a relative accuracy of $8 \%$.

\section{Spectral disentangling and preliminary physical properties of components $\mathrm{A}$ and $\mathrm{Ba}$}

Keeping the mean elements of Table 5 fixed, we used KOREL to disentangle the line profiles of components $\mathrm{A}$ and $\mathrm{Ba}$ in all six spectral regions. Synthetic spectra were then fitted to the disentangled profiles in order to estimate the effective temperatures $T_{\text {eff }}$, gravitational acceleration $g$, projected rotational velocity $v \sin i$, fractional luminosity $L_{\mathrm{R}}, R V$ and metallicity $Z$ of the
Table 4. FOTEL orbital solution for the triple system HD 152246.

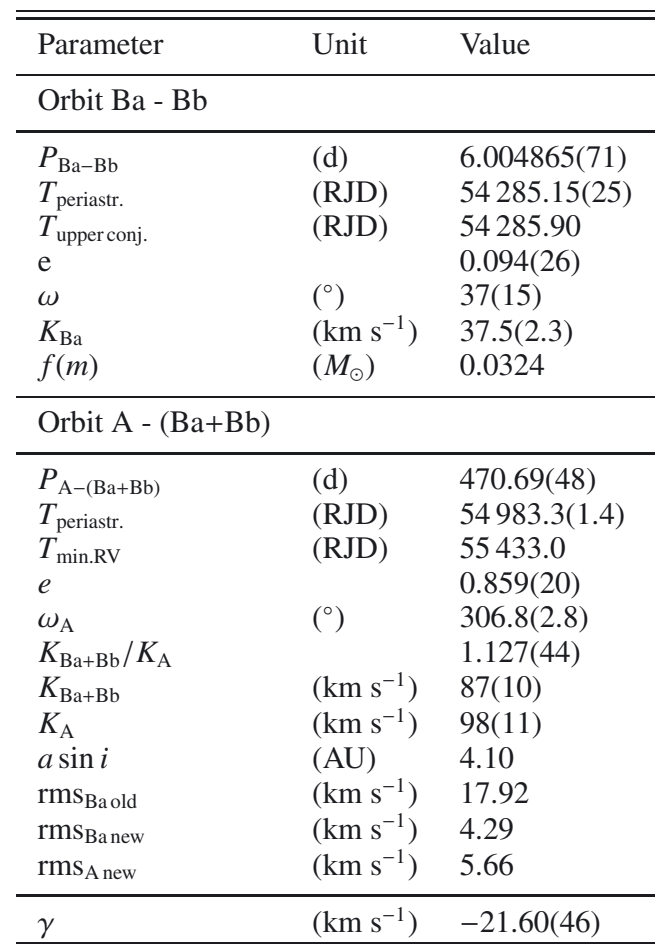

Notes. $a$ is the semi-major axis of the $470 \mathrm{~d}$ orbit.



Fig. 4. Astrometric orbital solution of the wide A, B pair (Table 6) and PIONIER interferometric measurements. The dashed line shows the line of nodes.

resolved components of the system. We attempted two independent approaches.

\subsection{TLUSTY model atmospheres and the OSTAR grid of synthetic spectra}

One of us (JN) has developed a programme, which interpolates in a pre-calculated grid of synthetic stellar spectra in the effective temperature, gravitational acceleration, and metallicity. The programme starts with an initial estimate of all the parameters to be optimised. The interpolated spectrum of each component is shifted in $\mathrm{RV}$, broadened to the projected rotational velocity with the programme ROTINS (written by Dr. I. Hubený) and 
Table 5. KOREL solutions for the triple star, $P=6.004865$ for the short orbit fixed.

\begin{tabular}{llllllll}
\hline \hline Element & He I 5876 & He II 5411 & C III/C IV region & O III 5592 & He I 6678 & He I 7065 & Mean \\
\hline short orbit & & & & & & & \\
\hline$T_{\text {peri. }}(\mathrm{RJD}-54285)$ & 0.338 & 0.296 & 0.350 & 0.366 & 0.356 & 0.356 & $0.344 \pm 0.025$ \\
$e$ & 0.1124 & 0.1122 & 0.1120 & 0.1123 & 0.1122 & 0.1123 & $0.1122 \pm 0.0001$ \\
$\omega_{\mathrm{Ba}}\left({ }^{\circ}\right)$ & 49.0 & 51.0 & 50.4 & 51.0 & 51.0 & 49.7 & $50.4 \pm 0.8$ \\
$K_{\mathrm{Ba}}\left(\mathrm{km} \mathrm{s}^{-1}\right)$ & 35.09 & 35.60 & 34.50 & 34.11 & 33.68 & 34.61 & $34.60 \pm 0.68$ \\
$f(m)\left(M_{\odot}\right)$ & 0.02538 & 0.02754 & 0.02507 & 0.02423 & 0.02328 & 0.02531 & $0.0251 \pm 0.0014$ \\
\hline long orbit & & & & & & & \\
\hline$P_{\text {long }}(\mathrm{d})$ & 469.91 & 469.90 & 469.98 & 469.88 & 470.00 & 470.00 & $469.95 \pm 0.05$ \\
$T_{\text {peri. }}(\mathrm{RJD}-54981)$ & 0.89 & 0.84 & 0.85 & 0.86 & 0.86 & 0.86 & $0.86 \pm 0.11$ \\
$e$ & 0.866 & 0.862 & 0.868 & 0.865 & 0.867 & 0.864 & $0.865 \pm 0.002$ \\
$\omega_{\mathrm{Ba}+\mathrm{Bb}}\left({ }^{\circ}\right)$ & 123.2 & 123.4 & 123.2 & 123.5 & 123.4 & 123.3 & $123.3 \pm 0.12$ \\
$K_{\mathrm{Ba}+\mathrm{Bb}}\left(\mathrm{km} \mathrm{s}{ }^{-1}\right)$ & 82.00 & 82.90 & 84.30 & 83.48 & 84.46 & 84.30 & $83.6 \pm 1.0$ \\
$K_{\mathrm{Ba}+\mathrm{Bb}} / K_{\mathrm{A}}$ & 0.8906 & 0.8985 & 0.9019 & 0.8915 & 0.8981 & 0.8978 & $0.8964 \pm 0.0044$ \\
$\left.K_{\mathrm{A}}(\mathrm{km} \mathrm{s})^{-1}\right)$ & 92.07 & 92.26 & 93.46 & 93.64 & 94.04 & 93.90 & $93.2 \pm 0.8$ \\
$a$ sin $i(\mathrm{AU})$ & 3.761 & 3.837 & 3.815 & 3.836 & 3.844 & 3.878 & $3.828 \pm 0.039$ \\
\hline
\end{tabular}

Table 6. Best-fit simultaneous RV and interferometric orbital solution of the HD 152246 A,B system.

\begin{tabular}{|c|c|c|c|}
\hline Parameter & Unit & A & B \\
\hline$P_{\text {out }}$ & [d] & \multicolumn{2}{|c|}{$470.54 \pm 0.53$} \\
\hline$a$ & [mas] & \multicolumn{2}{|c|}{$2.620 \pm 0.064$} \\
\hline$a$ & {$[\mathrm{AU}]$} & \multicolumn{2}{|c|}{$4.15 \pm 0.10$} \\
\hline$a_{j}(j=\mathrm{A}, \mathrm{B})$ & [mas] & $1.387 \pm 0.047$ & $1.234 \pm 0.038$ \\
\hline$a_{j}(j=\mathrm{A}, \mathrm{B})$ & {$[\mathrm{AU}]$} & $2.198 \pm 0.075$ & $1.955 \pm 0.060$ \\
\hline$e$ & & \multicolumn{2}{|c|}{$0.843 \pm 0.024$} \\
\hline$\omega_{\mathrm{A}}$ & {$\left[{ }^{\circ}\right]$} & \multicolumn{2}{|c|}{$303.71 \pm 2.95$} \\
\hline$T$ & [RJD] & \multicolumn{2}{|c|}{$54983.51 \pm 1.53$} \\
\hline$\gamma$ & {$\left[\mathrm{km} \mathrm{s}^{-1}\right]$} & \multicolumn{2}{|c|}{$-21.48 \pm 0.98$} \\
\hline$M_{\mathrm{A}} / M_{\mathrm{B}}$ & & \multicolumn{2}{|c|}{$0.893 \pm 0.038$} \\
\hline$i$ & {$\left[{ }^{\circ}\right]$} & \multicolumn{2}{|c|}{$112.46_{-9}^{+6.98}$} \\
\hline$\Omega$ & {$\left[{ }^{\circ}\right]$} & \multicolumn{2}{|c|}{$28.05_{-6.08}^{+7.68}$} \\
\hline$K$ & {$\left[\mathrm{~km} \mathrm{~s}^{-1}\right]$} & $87.27 \pm 10.4$ & $77.95 \pm 9.5$ \\
\hline$M$ & {$\left[M_{\odot}\right]$} & $20.35 \pm 1.50$ & $22.78 \pm 1.82$ \\
\hline$\chi_{\text {red }}^{2}$ & & \multicolumn{2}{|c|}{0.83} \\
\hline
\end{tabular}

Notes. $a_{\mathrm{A}}$ and $a_{\mathrm{B}}$ denote the separations of components A and B from the common centre of gravity $\left(a=a_{\mathrm{A}}+a_{\mathrm{B}}\right) . \omega_{\mathrm{A}}$ refers to the direction from the centre of mass to $\mathrm{A}$ at periastron.

multiplied by the fractional luminosity of the modelled component. The synthetic spectrum is compared to the disentangled one (normalised to the common continuum of the system) and the initial parameters are optimised by minimisation of $\chi^{2}$ (defined by Eq. (1) below) until best match is achieved:

$\chi^{2}=\sum_{i=1}^{\mathrm{N}} w_{\mathrm{i}}\left[I_{\mathrm{i}}^{\mathrm{disen}}-I_{\mathrm{i}}^{\mathrm{synt}}\left(T_{\mathrm{eff}}, g, v_{\mathrm{R}} \sin i, L_{\mathrm{R}}, \mathrm{RV}, Z\right)\right]^{2}$.

Here $N$ is the number of disentangled components of the system, $I_{i}^{\text {disen }}$ the disentangled spectrum, $I_{\mathrm{i}}^{\text {synt }}$ the synthetic spectrum and $w_{\mathrm{i}}$ the weight of the $i$-th component. Fractional luminosities must satisfy the condition that $\sum_{i=1}^{\mathrm{N}} L_{\mathrm{R}, \mathrm{i}}=1$ and metallicities must satisfy the condition $Z_{i}=Z_{1}$, for $i=1 \ldots N$. The $\chi^{2}$ is minimised using the downhill simplex method (Nelder \& Mead 1965).

All the weights of the disentangled spectra were set to one and the interpolation was carried out in the OSTAR grid
Table 7. Results of the best fit of the OSTAR grid of synthetic spectra to the observed ones.

\begin{tabular}{lcc}
\hline \hline Quantity & Component A & Component Ba \\
\hline$T_{\text {eff }}(\mathrm{K})$ & $32506 \pm 23$ & $32544 \pm 48$ \\
$\log \left(g_{[\mathrm{cgs}]}\right)$ & $3.738 \pm 0.014$ & $3.706 \pm 0.009$ \\
$v \sin i\left(\mathrm{~km} \mathrm{~s}^{-1}\right)$ & $200.4 \pm 2.9$ & $61.41 \pm 0.47$ \\
$L_{\mathrm{R}}$ & $0.5712 \pm 0.0021$ & $0.4288 \pm 0.0021$ \\
$\mathrm{RV}\left(\mathrm{km} \mathrm{s}^{-1}\right)$ & $-20.2 \pm 2.6$ & $-19.72 \pm 0.36$ \\
$Z\left(\mathrm{Z}_{\odot}\right)$ & 1.00 & 1.00 \\
\hline
\end{tabular}

Notes. The optimised parameters are: the effective temperature $T_{\text {eff }}$, gravitational acceleration $g$, projected rotational velocity $v_{\mathrm{R}} \sin i$, fractional luminosity $L_{\mathrm{R}}, \mathrm{RV}$, and metallicity $Z$.

(Lanz \& Hubeny 2003) based on TLUSTY model atmopspheres (Hubeny \& Lanz 1995). Several short segments covering the neighbourhood of the spectral lines mentioned in Table 5 were optimised simultaneously and for both components at once, the C III $5696 \AA$ and the C IV 5801 and $5811 \AA$ lines being treated as two separate data segments (not as one long segment as in KOREL). The results of minimisation of Eq. (1) are summarised in Table 7. To estimate uncertainties of all derived parameters, we repeated the optimisation of $\chi^{2}$ hundred times, each time starting the optimisation from a random point. The solutions having the final $\chi^{2}$ less than 1.06 times higher than the $\chi^{2}$ of the best solution were used to estimate the errors. The largest source of the error, uncertainty in the spectra (re)normalisation of the disentangled profiles, was not taken into account ${ }^{2}$. This means that the errors quoted in Table 7 should be taken as lower limits.

On modelling side, we note that TLUSTY models are static plane-parallel models with full non-LTE metal line blanketing. They are suitable for $\mathrm{O}$ stars that have weak stellar winds.

While the general fit of the disentangled by synthetic spectra resulting from the final solution of Table 7 was quite satisfactory, deviations up to several per cent of the continuum level were found in the cores of several investigated spectral lines.

2 Since the disentangling in KOREL is carried out in Fourier space, it happens in almost every practical application that the resulting disentangled spectra after inverse Fourier transformation have slightly warped continua and need some re-normalisation. 


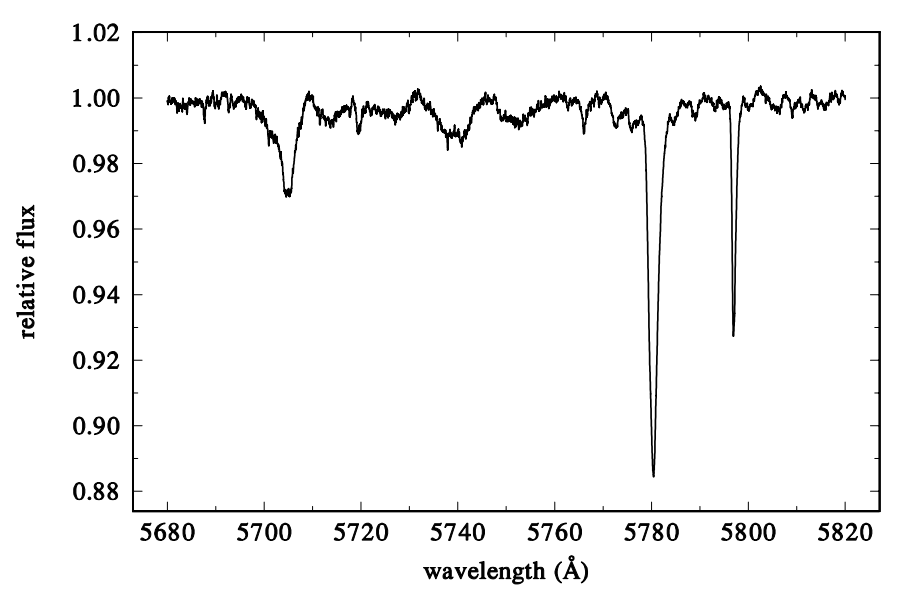

Fig. 5. Disentangled spectrum of diffuse interstellar bands in the 5680-5820 A.

There is a pronounced disagreement between the disentangled and synthetic spectra for the C III $5696 \AA$ line. The observed line is in emission in component $\mathrm{Ba}$. According to the synthetic spectra, this line should be in emission only at low $\log g$. When we tried to fit this line, and simultaneously also He II and He I lines, we ended with a strong disagreement with reality. However, we note that the presence of the $5696 \AA$ emission is common among main-sequence stars. The case of $15 \mathrm{Mon}$ (O7V) is known (Wilson 1955); see the ELODIE spectrum (Moultaka et al. 2004). By chance, we found that the line is in emission also in several O8 V stars: HD 161853 (FEROS spectrum) or in HD 49149 and HD 46966 (ELODIE spectra).

We also note that He I $6678 \AA$ line, and the two C IV lines for both, $\mathrm{A}$ and $\mathrm{Ba}$ component are stronger than suggested by the synthetic model but this difference is only on the level of $1 \%$ of the continuum level only. Once again, at least for He I line, such a behaviour is not unusual and has been observed for two other O stars, SZ Cam (Lorenz et al. 1998), and V1331 Aql (Lorenz et al. 2005).

It would be worth of investigation whether the observed deviations are indicative of a temperature stratification in the extended atmospheres and/or winds around the objects in question. For those colleagues, who study the diffuse interstellar bands, we also plot in Fig. 5 the disentangled spectrum of diffuse interstellar bands in the region from 5680 to $5820 \AA$, normalised to the joint continuum of the system.

\subsection{FASTWIND model spectra}

We also attempted to adjust the disentangled spectra with the FASTWIND model atmosphere (Puls et al. 2005; Rivero González et al. 2012) using an automatic fitting method (Mokiem et al. 2005; Tramper et al. 2011). In contrast to TLUSTY this programme uses spherical model atmospheres, non-LTE with line blanketing and models also stellar wind lines. On the other hand, FASTWIND only computes $\mathrm{H}$ and He profiles, i.e. there is no information for the C III $5696 \AA$ line. The atmospheres are not truly hydrodynamic, however, as the programme does not solve for the wind structure but adopts a wind law. Accurate mass loss estimates from the optical require recombination lines, like $\mathrm{H} \alpha$ or He II $4686 \AA$ which have not yet been analysed in the current investigation. The results of the fit are summarised in Table 8 .
Table 8. Results of the best fit of the FASTWIND grid of synthetic spectra to the observed ones.

\begin{tabular}{lcc}
\hline \hline Quantity & Component A & Component Ba \\
\hline$L_{\mathrm{R}}($ adopted $)$ & 0.5712 & 0.4288 \\
$T_{\text {eff }}(\mathrm{K})$ & 33500 & 34650 \\
$\log \left(g_{[\mathrm{cgs}]}\right)$ & 3.7 & 3.7 \\
$v \sin i\left(\mathrm{~km} \mathrm{~s}^{-1}\right)$ & 218 & 68 \\
$v_{\text {turb. }}\left(\mathrm{km} \mathrm{s}^{-1}\right)$ & 17 & 18 \\
$v_{\infty}\left(\mathrm{km} \mathrm{s}^{-1}\right)$ & 2161 & 1902 \\
$\log \dot{M}$ & -6.5 & -6.5 \\
$\beta$ & 1.2 & 0.95 \\
$R\left(R_{\odot}\right)$ & 9.3 & 7.9 \\
\hline
\end{tabular}

Notes. Parameter $\beta$ is the usual coefficient of the wind velocity law.

A detailed fit of some spectral line was not perfect in this case either.

More generally, it is obvious that a really reliable line profile modelling awaits further improvements, both on the theoretical and observational side. For instance, Massey et al. (2013) compared FASTWIND and independent CMFGEN model spectra with observed spectra of $10 \mathrm{O}$ stars and noted that the surface gravities of FASTWIND are systematically lower by 0.12 dex compared to CMFGEN and that both programmes have some problems to fit the He I lines for higher luminosity stars. Concerning the observed spectra, we believe that the spectral disentangling will provide better line profiles of both visible stellar components after we obtain good spectra during the periastron passage in the outer orbit.

\subsection{Preliminary physical properties of components $\mathrm{A}$ and $\mathrm{Ba}$}

Considering the above exercises, we warn against taking the results of Tables 7 and 8 too seriously at the present stage of investigation and postpone a more accurate determination of the physical properties of the system for a future study. For the moment, we consider reasonable to adopt $T_{\text {eff }}=33000 \pm 500$ and $v \sin i$ $=210 \pm 10 \mathrm{~km} \mathrm{~s}^{-1}$ for component A, and $T_{\text {eff }}=33600 \pm 600$ and $v \sin i=65 \pm 3 \mathrm{~km} \mathrm{~s}^{-1}$ for component $\mathrm{Ba}$.

\section{Physical properties of the system}

According to Sota et al. (2014) the integrated spectral type of HD 152246 is O9 IV, and according to Mermilliod \& Mermilliod (1998) $V=7 \mathrm{~m} .308, B-V=0$. 169 . For that spectral type, the expected $(B-V)_{0}=-0.30$, therefore the excess $E(B-V)=0.47$. HD 152246 is close to NGC 6231 (angular separation of about $\left.45^{\prime}\right)$. Sung et al. (2013) gives for this cluster $R=3 \mathrm{~m} .20$ and a distance modulus of $11.0(d=1585 \mathrm{pc})$. The systemic velocity of NGC 6231 is $-27.28 \pm 2.98$ as obtained from 10 high-mass stars (Sana et al. 2008); the scatter of individual RVs complies with the systemic velocity of HD 152246 as given in Table 6 corroborating its membership in Sco OB 1 association.

Moreover, Sana et al. (2005) derived basic physical properties of the massive spectroscopic and eclipsing binary CPD-41 $1^{\circ} 7742$, a member of NGC 6231 for which they were able to derive the distance modulus of $10.92 \pm 0.16$, in agreement with the above value. Applying the colour excess and a distance modulus of 11.0 to HD 152246 , we obtain $V_{0}=5.80$ and $M_{V}=-5$. 20 .

The fitting of the disentangled spectra yields the monochromatic flux ratio between the narrow-lined component $\mathrm{Ba}$ and 


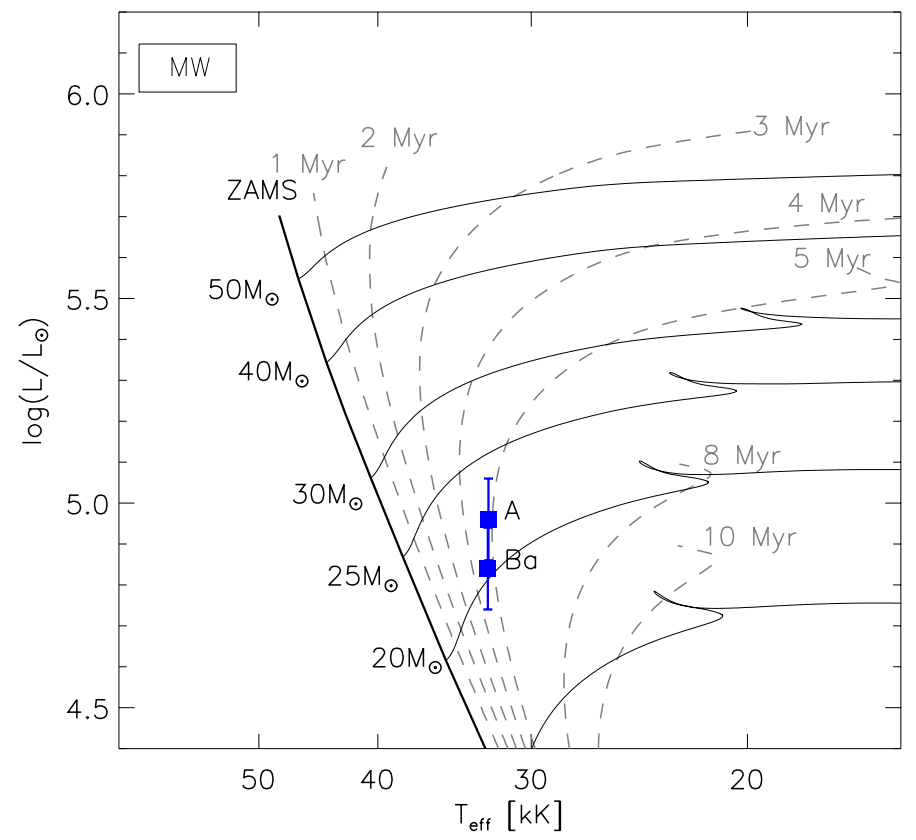

Fig. 6. Position of HD 152246 A and Ba in the HRD. Evolutionary tracks and isochrones of Brott et al. (2011) with initial rotation rate of $100 \mathrm{~km} \mathrm{~s}^{-1}$ are overlaid.

broad-lined component A as $0.429 / 0.571=0.751$. This is close to the ratio 0.77 found in the $H$ band by PIONIER (Table 3 ). However, we already noted that in this case the disentangling might not be reliable. Therefore, we prefer the value 0.77 ; the corresponding magnitude difference is 0.284 . Then the magnitude $M_{V}$ of the A component is -4 . 58 , of the B component -4 m.30. Martins et al. (2005) has $M_{V}=-4$. 05 for $09 \mathrm{~V}$ and -5 . 25 for O9 III; the agreement with the expected magnitude of component $\mathrm{A}$ (O9 IV) is a very good one. Component $\mathrm{Ba}$ is then $\mathrm{O} 9 \mathrm{~V}$.

The typical expected mass for HD $152246 \mathrm{~A}$, based on its spectral type, is $\sim 20 M_{\odot}$ (Martins et al. 2005), in agreement with the derived dynamical mass. Since the expected mass for an $\mathrm{O} 9 \mathrm{~V}$ star is $17.5 M_{\odot}$, the secondary $\mathrm{Bb}$ should contribute by about $6 M_{\odot}$; the mass ratio of 0.3 between $\mathrm{Bb}$ and $\mathrm{Ba}$ is quite acceptable given the SB1 nature of HD 152246B (the luminosity of the secondary can be neglected in the discussion made above).

\section{Discussion}

\subsection{Evolutionary status}

We used the BONNSAI ${ }^{3}$ Bayesian tool (Schneider et al. 2014) to compare the observational constraints and the single massive star evolutionary models of Brott et al. (2011). We used a Saltpeter IMF and flat priors for the age and initial rotational velocity.

For the A component, we provided the observed $T_{\text {eff }}$, $\log L_{\mathrm{bol}} / L_{\odot}, \log g, v \sin i$ and mass, together with their uncertainty. BONNSAI is able to find a set of models that perfectly reproduce the observed values. These correspond to initial masses of $21.4_{-0.9}^{+1.4} M_{\odot}$ and age of $4.7 \pm 0.3 \mathrm{Myr}$.

For the $\mathrm{Ba}$ component, we provided the same parameters with the exception of the stellar mass, that is not directly constrained by our observations owing to the uncertain mass of the

\footnotetext{
3 The BONNSAI web-service is available at http://www.astro. uni-bonn.de/stars/bonnsai
}

$\mathrm{Bb}$ component. No model was able to reproduce all parameters. The main issue was that our derived $\log g$ is too low. We removed the observational constraint on the gravity and re-ran BONNSAI. The set of models able to reproduce our observations indicate $\log g=4.03 \pm 0.15$, a present day mass of $18.4_{-1.1}^{+2.0}$ and an age of $4.8_{-1.5}^{+0.4} \mathrm{Myr}$. The agreement of the evolutionary mass of component $\mathrm{Ba}$ with the expected one based on its spectral properties is excellent. Similarly the evolutionary ages of both $\mathrm{A}$ and $\mathrm{Ba}$ component are identical, suggesting a coeval formation of the system. The location of both components in the Hertzsprung-Russell diagram (HRD) is displayed in Fig. 6. The derived age of HD 152246 agrees with that of other high-mass stars in NGC 6231 although the latter ones are model-dependent (Sung et al. 2013). If the rotating models of Ekström et al. (2012) are adopted the age is 4.0-7.0 Myr.

\subsection{Rotational velocities and dynamical status}

Intriguingly, the very similar $\mathrm{A}$ and $\mathrm{Ba}$ components in HD 152246 presents very different projected rotational velocities of 200 and $60 \mathrm{~km} \mathrm{~s}^{-1}$. Assuming the rotation and orbital spins of the wide binary are aligned, we would derive very similar values of 220 and $65 \mathrm{~km} \mathrm{~s}^{-1}$.

However, given the masses of the $\mathrm{Ba}, \mathrm{Bb}$ system are relatively well constrained to about 18.4 and $4.4 M_{\odot}$, we can use the FOTEL orbital solution of Table 4 to estimate the orbital inclination of the $\mathrm{Ba}, \mathrm{Bb}$ pair. We obtain $i_{\mathrm{Ba}, \mathrm{Bb}} \approx 32^{\circ}$. Assuming this time that rotation axis of the $\mathrm{Ba}$ component is aligned with the orbital spin of the short period system, we then obtain $v_{\text {rot,Ba }} \approx 120 \mathrm{~km} \mathrm{~s}^{-1}$, i.e. still significantly lower that the one of the A component.

BONNSAI best models indicate a probable radius of $6.75_{-1.20}^{+1.72} R_{\odot}$ for the $\mathrm{Ba}$ component. Tidal synchronisation of $\mathrm{Ba}$ in the $\mathrm{Ba}, \mathrm{Bb} 6 \mathrm{~d}$ orbit would result in rotational velocity of $57_{-10}^{+14} \mathrm{~km} \mathrm{~s}^{-1}$. Such velocity is in agreement with the measured projected rotational velocity and would suggest an inclination close to $90^{\circ}$ for the spin axis of HD $152246 \mathrm{Ba}$. If this scenario is correct, then it would mean that the spin and orbital axis are not aligned in the $\mathrm{Ba}, \mathrm{Bb}$ system. One might also speculate whether the star formation process had not allotted similar amounts of angular momentum to both components $\mathrm{A}$ and $\mathrm{B}$. If so, this resulted in fast spin for A and the creation of a binary star in B.

\section{Conclusions}

Several conclusions can be drawn here:

i. the evolutionary ages of the O stars in HD 152246 suggest a co-eval formation of the triple system;

ii. with orbital inclination $i_{\mathrm{A}, \mathrm{B}} \approx 112^{\circ}$ and $i_{\mathrm{Ba}, \mathrm{Bb}} \approx 30\left(\right.$ or $\left.150^{\circ}\right)$ the two orbits are not co-aligned;

iii. the components $\mathrm{A}$ and $\mathrm{Ba}$ likely have different rotational velocities;

iv. the component $\mathrm{Ba}$ is possibly synchronised, but if this is correct the spin and orbital axis are not aligned;

v. HD 152246 A,B pair has an eccentricity significantly higher than expected from the period-eccentricity diagram of other O-type systems (Sana et al. 2012).

It is possible that several of these properties result from the hierarchical nature and extreme eccentricity of the system. In this scenario, each close encounter of the $\mathrm{Ba}, \mathrm{Bb}$ pair with the $\mathrm{A}$ component would perturb the $\mathrm{Ba}, \mathrm{Bb}$ inclination and spin alignments. Even if the A and Ba components were born identical, the 
regular interactions between the three bodies may result in different rotation velocities. Alternatively, HD 152246 may be born as hierarchical quadruple systems formed by two close-binaries. The Aa, Ab initial binary may have been driven into coalescence because of stellar evolution (if the initial period was extremely short) or dynamical interaction with the $\mathrm{Ba}, \mathrm{Bb}$ pair. This may have lead to a catastrophic event that may have pumped up the eccentricity of the wide system and left a rapidly rotating A component. Such event should however have happened early in the evolutionary life of HD 152246 as no rejuvenation effect is seen in the HRD. Future abundance determination of the HD 152246 components may help provide further constraints on these scenarios.

Acknowledgements. This publication is supported as a project of the NordrheinWestfälische Akademie der Wissenschaften und der Künste in the framework of the academy programme by the Federal Republic of Germany and the state Nordrhein-Westfalen. The research of P.H., P.M., and J.N. was supported by the grant P209/10/0715 of the Czech Science Foundation and from the research programme MSM0021620860. We are grateful for the help of A. Barr Domínguez, K. Fuhrmann, L. Kaderhandt and M. Ramolla during the observations and the reduction. We thank Universidad Católica del Norte in Antofagasta, Chile, for continuous support. The use of the NASA/ADS bibligraphical service and SIMBAD electronic database are gratefully acknowledged. PIONIER is funded by the Université Joseph Fourier (UJF), the Institut de Planétologie et d'Astrophysique de Grenoble (IPAG), the Agence Nationale pour la Recherche (ANR-06-BLAN-0421 and ANR-10-BLAN-0505), and the Institut National des Sciences de l'Univers (INSU PNPand PNPS).The integrated optics beam combiner result from a collaboration between IPAG and CEA=LETI based on CNRS R\&T funding.

\section{References}

Brott, I., de Mink, S. E., Cantiello, M., et al. 2011, A\&A, 530, A115 Chini, R., Hoffmeister, V. H., Nasseri, A., et al. 2012, MNRAS, 424, 1925 Conti, P. S., \& Ebbets, D. 1977, ApJ, 213, 438

Conti, P. S., Leep, E. M., \& Lorre, J. J. 1977, ApJ, 214, 759

Dachs, J., Kaiser, D., Nikolov, A., Sherwood, W. A. 1982, A\&AS, 50, 261 Ekström, S., Georgy, C., Eggenberger, P., et al. 2012, A\&AS, 537, 146

Fuhrmann, K., Chini, R., Hoffmeister, V. H., et al. 2011, MNRAS, 411, 2311

Grigsby, J. A., Morrison, N. D., \& Anderson L. S. 1992, ApJS, 78, 205

Garrison, R. F., Schild, R. E., \& Hiltner, W. A. 1977, ApJS, 35, 111
Hadrava, P. 2004a, Publ. Astron. Inst. Acad. Sci. Czech Rep., 92, 1 Hadrava, P. 2004b, Publ. Astron. Inst. Acad. Sci. Czech Rep., 92, 15 Haguenauer, P., Abuter, R., Alonso, J., et al. 2008, in SPIE Conf. Ser., 7013, 11 Haguenauer, P., Alonso, J., Bourget, P., et al. 2010, in SPIE Conf. Ser., 7734, 3 Harmanec, P. 1998, A\&A, 335, 173

Heske, A., \& Wendker, H. J. 1984, A\&AS, 57, 205

Hog, E., Fabricius, C., Makarov, V. V., et al. 2000, A\&A, 355, L27

Howarth, I. D., Siebert, K. W., \& Hussain, G. A. J. 1997, MNRAS, 284, 265

Hubeny, I., Lanz, T. 1995, ApJ, 439, 875

Klare, G., \& Neckel, T. 1977, A\&AS, 27, 215

Lanz, T., \& Hubeny, I. 2003, ApJS, 146, 417

Le Bouquin, J.-B., Berger, J.-P., Lazareff, B., et al. 2011, A\&A, 535, A67

Lorenz, R., Mayer, P., \& Drechsel, H. 1998, A\&A, 332, 909

Lorenz, R., Mayer, P., \& Drechsel, H. 2005, MNRAS, 360, 915

Martins, F., Schaerer, D., \& Hillier, D. J. 2005, A\&A, 436, 1049

Mason, B. D., Gies, D. R., Hartkopf, W. I., et al. 1998, AJ, 115, 821

Massey, P., Neugent, F., Hillier, D. J., Puls, J. 2013, ApJ, 768, 6

Mayer, P., Harmanec, P., \& Pavlovski, K. 2013, A\&A, 550, A2

Mermilliod, J.-C., \& Mermilliod, M. 1998, Online catalogue of $U B V$ photometry http://obswww . unige.ch/gcpd/cgi-bin/photoSys.cgi

Mokiem, M. R., de Koter, A., Puls, J., et al. 2005, A\&A, 441, 711

Morgan, W. W., Code, A. D., \& Whitford, A. E. 1955, A\&A, 441, 711

Moultaka, J., Ilovaisky, S. A., Prugniel, P., \& Soubiran, C. 2004, PASP, 116, 693

Nasseri, A., Chini, R., Harmanec, P., et al. 2013, EAS Pub. Ser., 64, 411

Nelder, J. A., \& Mead, R. 1965, Comput. J., 7, 308

Penny, L. R. 1996, ApJ, 463, 737

Perryman, M. A. C., \& ESA 1997, The HIPPARCOS and TYCHO catalogues, ESA SP Ser. 1200 (Noordwijk: ESA Publ. Division)

Puls, J., Urbaneja, M. A., Venero, R., et al. 2005, A\&A, 435, 669

Rivero González, J. G., Puls, J., Najarro, F., Brott, I. 2012, A\&A, 537, A79

Sana, H., Antokhina, E., Royer, P., et al. 2005, A\&A, 441, 213

Sana, H., Gosset, E., Nazé, Y., et al. 2008, MNRAS, 386, 447

Sana, H., de Mink, S. E., de Koter, A., et al. 2012, Science, 337, 444

Sana, H., Le Bouquin, J.-B., Mahy, L., et al. 2013, A\&A, 553, A131

Sana, H., Le Bouquin, J.-B., Lacour, S., et al. 2014, ApJS, submitted

Schild, R. E., Hiltner, W. A., \& Sanduleak, N. 1969, ApJ, 156, 609

Schild, R. E., Garrison, R. F., \& Hiltner, W. A. 1983, ApJS, 51, 321

Schneider, F. R. N., Langer, N., de Koter, A., et al. 2014, A\&A, in press DOI: $10.1051 / 0004-6361 / 201424286$

Sota, A., Maíz Apellániz, J., Morrell, N. I., et al. 2014, ApJS, 211, 10

Stickland, D. J., \& Lloyd, C. 2001, The Observatory, 121, 1

Sung, H., Sana, H., \& Bessell, M. S. 2013, AJ, 145, 37

Thackeray, A. D., Tritton, S. B., \& Walker, E. N. 1973, Mem. Roy. Astron. Soc., 77, 199

Tramper, F., Sana, H., de Koter, A., \& Kaper, L. 2011, ApJ, 741, L8

Walborn, N. R. 1973, AJ, 78, 1067

Wilson, R. 1955, The Observatory, 75, 222 